\title{
Nonlinear Absorbing-Loop Mirror in a Holmium-Doped Fiber Laser
}

\author{
Junqing Zhao, Member, OSA, Jian Zhou, Yuyuan Jiang, Lei Li, Deyuan Shen, Andrey Komarov, Lei Su, \\ Dingyuan Tang, Member, OSA, Mariusz Klimczak, and Luming Zhao, Senior Member, IEEE
}

\begin{abstract}
Nonlinear amplifying-loop mirror (NALM) and nonlinear optical loop mirror (NOLM) are two basic Sagnac interferometers widely used in mode-locked fiber lasers. Here we construct a novel variant by replacing the amplifier in an NALM with an absorber, which forms a type of nonlinear absorbing-loop mirror (NAbLM). The optical waves counter-propagating within an ideal NAbLM possess equal optical powers when they return and interfere inside the used fiber coupler owing to the equal absorptions. Meanwhile, their nonlinear phase shifts can still differ if an asymmetric distribution of the absorber is employed. We theoretically predict that, in comparison to typical NALM and NOLM, the NAbLM can achieve relatively higher extinction ratio and larger modulation depth, resting on the unique equal-power interference. We then first used it as a saturable absorber in a holmium-doped fiber laser and achieved passively mode-locked operations in both normal and anomalous dispersion regimes. In the normal dispersion regime, $h$-shaped pulse was generated; whereas in the anomalous dispersion regime, both noise-like and dissipative-soliton-resonance-like pulses could be produced. NAbLM enables more options in nonlinear fiber optics especially when a high extinction ratio or large modulation depth is required.
\end{abstract}

Index Terms-Fiber devices, Interferometers, Fiber lasers.

\section{Manuscript received.}

This work was supported in part by the National Natural Science Foundation of China (NSFC) under Grants 61705094, 11911530083, 11674133, and 61575089 , in part by the Natural Science Foundation of Jiangsu Province under Grant BK20170243, in part by the Russian Foundation for Basic Research (RFBR) under Grant 19-52-53002, in part by the Royal Society under Grant IE161214, in part by the Protocol of the 37th Session of China-Poland Scientific and Technological Cooperation Committee under Grant 37-17, and in part by the European Union's Horizon 2020 research and innovation programme under the Marie Skłodowska-Curie Grant 790666. (Corresponding author: Luming Zhao)

J. Zhao, J. Zhou, Y. Jiang, L. Li, D. Shen, and L. Zhao are with the Jiangsu Key Laboratory of Advanced Laser Materials and Devices, Jiangsu Collaborative Innovation Center of Advanced Laser Technology and Emerging Industry, School of Physics and Electronic Engineering, Jiangsu Normal University, Xuzhou 221116, Jiangsu, China (e-mail: junqingzhao@outlook.com; 835139040@qq.com; 1461673357@qq.com; Leelei@jsnu.edu.cn; shendy@fudan.edu.cn; lmzhao@ieee.org).

A. Komarov is with the Institute of Automation and Electrometry, Russian Academy of Sciences, Academician Koptyug Prospekt1, 630090 Novosibirsk, Russia (e-mail: andnsk@iae.nsk.su).

L. Su is with the School of Engineering and Materials Science, Queen Mary University of London, London, UK (email: 1.su@qmul.ac.uk).

D. Tang is with the School of Electrical and Electronic Engineering, Nanyang Technological University, Singapore (e-mail: edytang@ntu.edu.sg).

M. Klimczak is with the Faculty of Physics, University of Warsaw, Warsaw, Poland, and also with the Institute of Electronic Materials Technology, Wolczynska 133, 01-919 Warsaw, Poland (e-mail: mklimcz@gmail.com).

\section{INTRODUCTION}

WING to the interesting nonlinear effects with fiber interferometers, different architectures have been explored for various applications. Nonlinear Sagnac interferometer is commonly considered as one of the most widely investigated devices. It possesses power-dependent optical switching due to the induced asymmetrical nonlinear phase shifts. Particularly, as a power-dependent device, it has been used as a kind of artificial saturable absorber (SA). The general framework is to asymmetrically introduce some nonlinear element in the Sagnac interferometer [1], for producing relative nonlinear phase difference $\Delta \varphi_{N L}$. It is typically accomplished by connecting a long piece of fiber to the two output ports of an unequally-split fiber coupler, which has been so-called as nonlinear optical loop mirror (NOLM) [2]. If an amplifier is asymmetrically incorporated in a loop mirror, a variant of NOLM, i.e., nonlinear amplifying-loop mirror (NALM) is formed [3]. This can considerably reduce the optical switching threshold owing to the $\Delta \varphi_{N L}$ amplification. As a particularly important application, most investigations on NOLM and NALM have been devoted to their usage in passively mode-locked fiber lasers [4]-[8].

Apart from NOLM and NALM, few other alternatives were also investigated for optical switching related purposes. Those include combination of a semiconductor optical amplifier (SOA) and an optical attenuator [9], loss-imbalanced NOLM with inclusion of some lumped loss [10, 11], NOLM with incorporation of a directional phase shifter [12], etc.

It is further noted that although a 50:50 fiber coupler can even be used in the NALM, the counter-propagating portions of laser pulses after amplifications are still not equal in peak power. This is based on the fact that the forward and backward gains are typically different in practice. Consequently, the resultant coherence is also not complete or thorough. Thus, only partial modulation and incomplete extinction can be achieved with whether NALM, NOLM, or other alternatives, owing to the unequal power coherence. To address this, in this paper, we construct another type of all-fiber nonlinear Sagnac interferometer, i.e., replacing the amplifier in a NALM with a fiber absorber, and name it as nonlinear absorbing-loop mirror (NAbLM). We compare the constructed NAbLM with both NOLM and NALM, theoretically predict its special properties, and then, as a preliminary application, check its viability as an artificial SA to enable passive mode-locking of a holmium-doped fiber (HDF) laser. 


\section{BASIC ARCHITECTURE AND THEORY OF NABLM}

Figure 1 schematically depicts the basic architectures of NOLM, NALM, and our proposed NAbLM, respectively, including the power flows as indicated (the thicker the red arrow, the higher the indicated power). To achieve $\Delta \varphi_{N L}$ between the counter-propagating directions, the NOLM employs a non-50:50 fiber coupler, whereas NALM/NAbLM employs a 50:50 fiber coupler and meanwhile asymmetrically incorporates a fiber amplifier/absorber. Although all the couplers will show asymmetry more or less in practice, here for simplicity we assume that a 50:50 symmetry coupler is possible, only in theory.

As for the interfering powers of the two counter-propagating optical waves at the fiber coupler, they are certainly different due to the use of a non-50:50 fiber coupler in the NOLM. As for an NALM, it has always been assumed that the two interfering, counter-propagating optical waves are the same in power when one calculates the related switching properties [2]. However, they are quite different in practice due to that the fiber laser/amplifier gain is always direction-dependent. Typically, the forward gain is considerably less than the backward gain [5] regarding to the pumping-launching direction. In Fig. 1(b), we assume that the forward and backward gains are along the clockwise and counterclockwise directions, respectively.

For our proposed NAbLM, however, as seen in Fig. 1(c), it can ideally guarantee equal powers of the two counter-propagating optical waves when they meet each other in the fiber coupler since the fiber absorption is naturally direction-independent. This is a significant difference from NOLM and NALM. Meanwhile, adequate $\Delta \varphi_{N L}$ can still be produced, since the two waves preserve power difference through most of the fiber loop due to the asymmetrically located fiber absorber.

We have also paid attention to another notable point. For either NOLM or NALM, there is some portion of power without coherence that will then be split again by the fiber coupler. One part will be reflected back to the input port, and the left will transmit into the output port. We use dashed red arrows to represent them, as seen in Figs. 1(a) and 1(b). As for our proposed NAbLM in Fig. 1(c), however, the equally-split portions together with the direction-independence of the fiber absorber will result that all the power can participate the coherence in the fiber coupler, i.e., a complete coherence is reached. For simplicity in demonstration of the switching characteristics for each loop mirror, here we only illustrate a particular situation, i.e., the two interfering power portions have already accumulated a phase difference of integer times of $\pi$. Thus, for our proposed NAbLM, all the power can be perfectly switched to the output port benefiting from the complete coherence. Whereas for the NOLM or the NALM, only the interfering power can be switched to the output port. Figures 1(a) through 1(c) show these characteristics clearly.

We will give some further analysis concerning our proposed NAbLM. For further gaining some theoretical insight and obtaining a general result, we assume that the splitting ratio of the used fiber coupler is $\rho(0<\rho<0.5)$, and the pulse peak power $P_{\text {peak }}$ is absorbed by a factor $\alpha$ when it propagates through the fiber absorber, i.e., $P_{\text {peak }}$ becomes $\alpha P_{\text {peak }}$.
In general, extinction ratio of any optical signal going through an optical device or system can be defined as

$$
E R=\frac{P_{\text {out }, \text { max }}}{P_{\text {out }, \text { min }}}
$$

As for the coherent properties of NAbLM, $P_{\text {out,max }}$ and $P_{\text {out,min }}$ represent the constructive and destructive pulse peak powers, respectively, depending on that $\Delta \varphi_{N L}$ is $2 n \pi$ or $(2 n+1) \pi,(n=0,1,2, \cdots)$. According to the basic theory of coherence, it can be easily deduced that

$\left\{\begin{array}{c}P_{\text {out }, \text { max }}=4 \alpha \rho P_{\text {peak }}+\alpha(1-2 \rho) P_{\text {peak }}, \Delta \varphi_{N L}=2 n \pi \\ P_{\text {out } \text { min }}=\alpha(1-2 \rho) P_{\text {peak }}, \Delta \varphi_{N L}=(2 n+1) \pi\end{array}\right.$

where $n=0,1,2, \cdots$. Thus,

$$
E R=\frac{1+2 \rho}{1-2 \rho}
$$

This relationship, as plotted in Fig. 1(d), indicates that the $E R$ in coherence radically surges towards infinity as $\rho \rightarrow 0.5$. Thus, NAbLM can reach a virtually high $E R$ due to its equal-power coherence, representing a significant enhancement in comparison to both NOLM and NALM. This calculation is consistent with the results with the physical model shown in Ref. [12], but, differently, herein gives a simplified but fundamental insight of NAbLM.

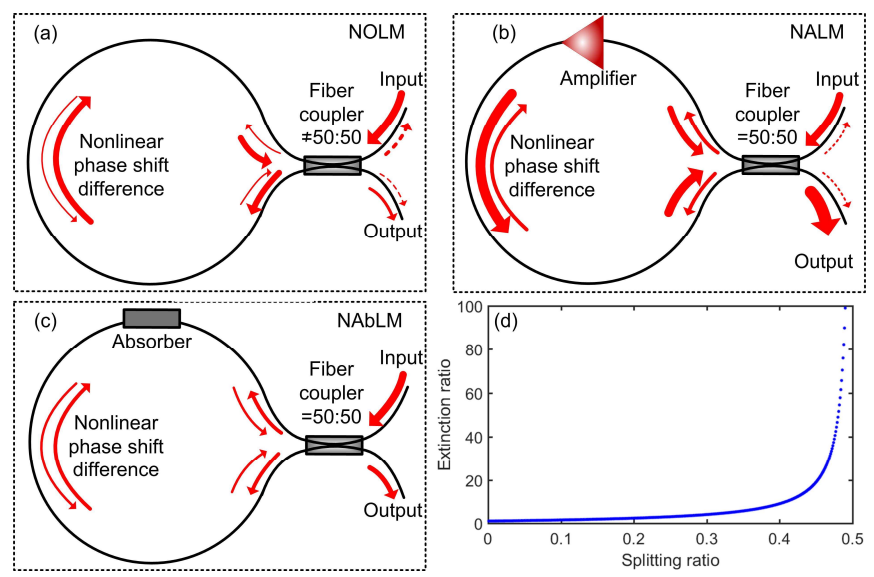

Fig. 1. Basic schematics of (a) NOLM, (b) NALM, and (c) NAbLM; (d) coherence extinction ratio of NAbLM as a function of splitting ratio.

We define $P_{0}=\left|E_{0}\right|^{2}$ as the total power of the two counter-propagating waves in the NAbLM after absorptions, and $P_{t}=\left|E_{t}\right|^{2}$ as the further transmitted power through the fiber coupler. $A_{0}$ and $A_{t}$ are the corresponding optical fields. Referring to typical NOLM [13], the transmittivity of the NAbLM is then

$T_{s} \equiv \frac{\left|E_{t}\right|^{2}}{\left|E_{0}\right|^{2}}=1-2 \rho(1-\rho)\{1+\cos [(1-\rho-$

$\left.\left.\alpha \rho) \frac{2 \pi n_{2}}{\lambda A_{\text {eff }}} P_{\text {peak }} L\right]\right\}$

where $n_{2}$ is the nonlinear-index coefficient of the used fiber, $\lambda$ is the light wavelength in vacuum, $A_{\text {eff }}$ is the effective mode area, and $P_{\text {peak }}$ is the pulse peak power. There are two paths connecting the fiber absorber and the fiber coupler, one shorter and the other longer. $L$ represents the distance that covers the longer one. The part $(1-\rho-\alpha \rho) \frac{2 \pi n_{2}}{\lambda A_{e f f}} P_{\text {peak }} L$ accounts for $\Delta \varphi_{N L}$. At low power, $\Delta \varphi_{N L} \rightarrow 0$. Thus,

Clearly

$$
T_{s}=1-2 \rho(1-\rho)\left[1+\cos \left(\Delta \varphi_{N L}\right)\right]
$$




$$
T_{S} \stackrel{\Delta \varphi_{N L} \rightarrow 0}{\longrightarrow} 1-4 \rho(1-\rho)
$$

Further, $T_{s}$ approaches 0 as $\rho \rightarrow 0.5$. In this case, no light can transmit through the NAbLM, i.e. the switching is off. At high peak power, the light can be fully switched from the input to the output port, i.e., the NAbLM becomes completely transmissive, whenever

$$
\Delta \varphi_{N L}=(2 n+1) \pi,(n=0,1,2, \cdots)
$$

For $\rho=0.5$ and $n=0$, the 1 st order switching peak power is reached, which also corresponds to the lowest fully-saturated power. We use $P_{\text {sat }}$ to represent the saturation power. $P_{\text {sat }}$ is defined as a power level that an optical device (here the NAbLM) becomes transparent or absorption-less once it is reached. Thus,

$$
P_{\text {peak }}=P_{\text {sat }}=\frac{\lambda A_{\text {eff }}}{(1-\alpha) n_{2} L}
$$

Thus, if the input power is not too high at first, the higher it is, i.e., the closer to $P_{\text {sat }}$, the greater the transmittivity of the NAbLM. From another point of view, this is just equivalent to saturable absorption. Thus, NAbLM can act as an aritificial SA.

Certainly, it can be further deduced that the transmittivity of the NAbLM becomes smaller as $P_{\text {peak }}$ increases from $P_{\text {sat }}$ to $2 P_{\text {sat }}$. Thus, now the NAbLM acts as a reverse saturable absorber (RSA), i.e., an optical limiter. It switches to be an artificial SA again when $P_{\text {peak }} \in\left[2 P_{\text {sat }}, 3 P_{\text {sat }}\right]$. In general, the NAbLM periodically switches between SA and RSA depending on $P_{\text {peak }}$,

$$
P_{\text {peak }} \in\left\{\begin{array}{c}
{\left[2 n P_{\text {sat }},(2 n+1) P_{\text {sat }}\right], S A,} \\
{\left[(2 n+1) P_{\text {sat }},(2 n+2) P_{\text {sat }}\right], R S A,}
\end{array}\right.
$$

where $n=0,1,2, \cdots$.

Again, owing to the virtually high $E R$ benefiting from the equal power coherence with the NAbLM, the modulation depth, either being used as SA or RSA, can approach $100 \%$. Thus, in theory (at least) the NAbLM should be able to achieve a higher $E R$ and larger modulation depth in comparison to both NOLM and NALM.

\section{EXPERIMENTAL CONFIGURATION}

Here, we take some preliminary investigations on the NAbLM using as an artificial SA in an HDF laser, as shown in Fig. 2(a). This laser includes a unidirectional ring and a bidirectional fiber loop, connected by a 50:50 fiber coupler. The bidirectional fiber loop asymmetrically incorporating a piece of unpumped HDF constitutes an NAbLM.

In the unidirectional ring, we employ a cascaded-pumping scheme that is similar to our previous design [5], for achieving low quantum loss. A distributed feedback laser diode (DFB-LD) with peak emission at $\sim 1570.4 \mathrm{~nm}$ and a followed erbium-ytterbium fiber amplifier (EYFA) constitute the pump source. The pump light is coupled into $\sim 2.35 \mathrm{~m}$ single-mode thulium-doped fiber (TDF) via a filter wavelength division multiplexer (FWDM, Lightcomm Technology Co., Ltd, China). The TDF has a specified absorption at $790 \mathrm{~nm}$ of $\sim 182 \mathrm{~dB} / \mathrm{m}$. The pump leakage is rejected with another FWDM. The emission from the TDF is then subsequently used to cascade-pump $\sim 2.1 \mathrm{~m}$ HDF with a specified absorption of $\sim 39$ $\mathrm{dB} / \mathrm{m}$ at $1150 \mathrm{~nm}$. An isolator enables the unidirectional propagation. An 18:82 fiber coupler provides the output. A squeezer-type fiber polarization controller (FPC-1) is used to manipulate the local polarization state.

To construct the NAbLM, a piece of unpumped HDF is used as the fiber absorber. According to our experimental measurement, the small-signal absorption factor is $\alpha \approx 0.746$ $\mathrm{m}^{-1}$. Thus, a longer HDF will result in a stronger absorption. Several pieces of ultra-high numerical aperture fiber (UHNA7, Nufern-Coherent, Co.) with a total length of $\sim 121.5 \mathrm{~m}$ are incorporated to enhance the accumulative nonlinearity. The local polarization state in the NAbLM is manipulated by using a three-paddle FPC (FPC-2) with looped UHNA7 fiber within. Since $\Delta \varphi_{N L}$ is accumulated mainly in the UHNA7 fiber, we use the $\Delta \varphi_{N L}$ in it to roughly estimate the overall switching characteristics of the NAbLM. As for the UHNA7 fiber, $n_{2} \approx$ $4.48 \times 10^{-20} \mathrm{~m}^{2} / W \quad, \quad A_{\text {eff }} \approx 1.416 \times 10^{-11} \mathrm{~m}^{2}, \quad L=$ $121.5 \mathrm{~m}$. Substituting these values into Eq. (4) and letting $\rho=$ 0.5 and $\lambda=2.05 \times 10^{-6} \mathrm{~m}$, we obtain

$$
T_{s}=0.5-0.5 \cos \left[0.589(1-\alpha) P_{\text {peak }}\right]
$$

Based on Eq. (10), we can roughly plot the optical switching properties of the HDF-based NAbLM at different absorption factors. As shown in Fig. 2(b), the more the absorbed optical wave (i.e., the smaller the $\alpha$ ), the higher the required peak power to reach the switching threshold or power saturation. Based on Eq. (8) and using the related parameter values, we can further plot the relationship between the saturation power $P_{\text {sat }}$ and absorption factor $\alpha$, as depicted in Fig. 2(c). As seen, $P_{\text {sat }}$ increases with $\alpha$, i.e., reversely increases with absorption. Thus, a high-efficiency absorber, i.e., a longer piece of HDF or higher-dopant HDF, is favorable to lower $P_{\text {sat }}$. However, they do not satisfy a linear relationship. Thus, a pre-evaluation on the used rare-earth-doped fiber would help to reach an appropriate compromise between $\alpha$ and $P_{s a}$.

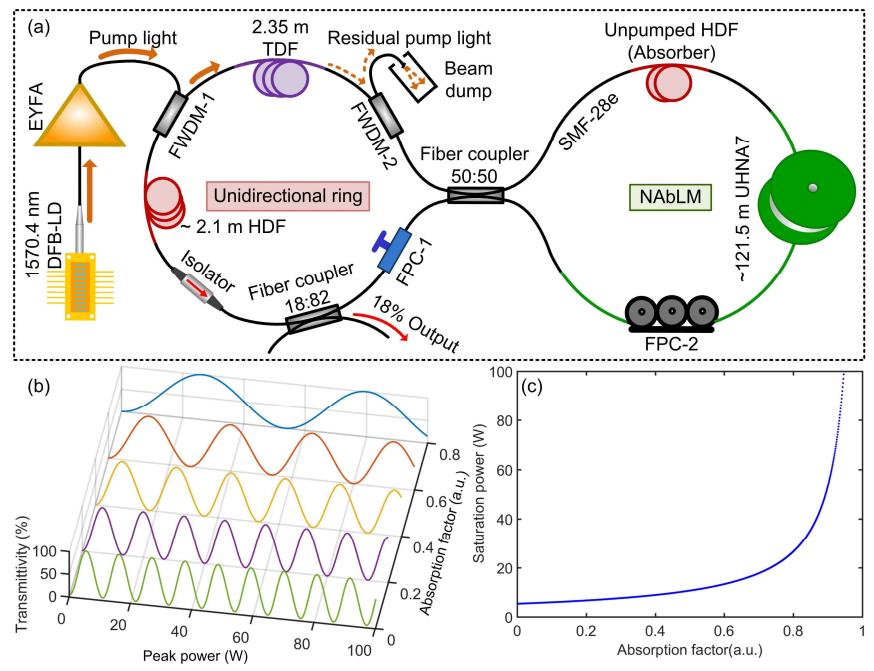

Fig. 2. (a) Schematic configuration of the NAbLM-incorporated HDF laser; (b) transmittivity of the NAbLM as a function of peak power at different absorption factors; (c) saturation power (the 1st order full-switching power) as a function of absorption factor.

\section{RESULTS AND DISCUSSION}

In experiment, without unpumped HDF, no pulse emission was observed no matter how we adjusted the two FPCs and varied the pump power. This also verified that the split optical 
waves were very close in power and no adequate $\Delta \varphi_{N L}$ could be induced although a long piece of UHNAF was incorporated in the loop. This could also be understood from Eq. (3) and Fig. 2(c): $\alpha \rightarrow 1, P_{\text {sat }} \rightarrow \infty$.

When $\sim 1$ m unpumped HDF was incorporated, stable single pulse was generated with an appropriately set polarization state and the launched pump power above $\sim 1.91 \mathrm{~W}$, indicating that an artificial SA, i.e. NAbLM, was formed. We measured the temporal traces by using an InGaAs photodetector (ET-5000, Electro-Optics Technology, Inc.) and a digital oscilloscope (DSO9104A, Agilent Technologies, Inc.). As plotted in Fig. 3(a), the emitted pulse showed h-shaped pulse profile, whose duration scaled with the pump power. This is similar to our previous results using NOLM [4], but here the peak of each pulse leading edge is less sharp comparatively, probably due to the leading edge suppression by the NAbLM's reabsorption.

We then changed the unpumped HDF length to check the absorption-related characteristics. With $\sim 1$ to $\sim 3$ m unpumped HDF, we could readily achieve the h-shaped pulse. However, beyond such a range it became difficult, which could be understood as follows. When the HDF is too short, the absorption is too weak, so the resulted peak power difference between the counter-propagating waves will be insufficient to produce enough $\Delta \varphi_{N L}$ in the NAbLM. Whereas, if the HDF is too long, the absorption becomes too strong. Consequently, both counter-propagating beams after the HDF will be too weak, again resulting in insufficient $\Delta \varphi_{N L}$. Thus, in general NAbLM is an input-related device. To make it functioning well as an SA (or RSA), appropriate absorbing fiber length should be chosen depending on the intensity of the input signal.

Figures 3(b) through 3(d) plot the output characteristics with unpumped HDF length of $\sim 1 \mathrm{~m}$ and $\sim 3 \mathrm{~m}$, respectively. The longer the incorporated HDF, the shorter and lower the emitted pulse, as shown in Fig. 3(b). The corresponding optical and radio frequency (RF) spectra were plotted in Figs. 3(c) and 3(d), respectively. The optical spectra were measured by using an optical spectrum analyzer (OSA, AQ6375B, Yokogawa Test \& Measurement Co.) with resolution of $0.05 \mathrm{~nm}$. The different spectral profiles, i.e. one with two peaks and the other with a single broadband $(\sim 6.68 \mathrm{~nm}$ at $3 \mathrm{~dB})$, might be caused by different filtering effects of the as-used NAbLMs. The RF traces were measured with the aforementioned photodetector and a radio frequency spectrum analyzer (N9320B, Agilent Technologies, Inc.) with resolution bandwidth of $10 \mathrm{~Hz}$. The peak RF was always determined by the cavity length. Signal-to-noise ratios (SNRs) in two cases were both greater than $55 \mathrm{~dB}$, indicating stable operations. We also noted that the maximum average output power lowered down from $\sim 38.3$ to $\sim 29.6 \mathrm{~mW}$, i.e., single pulse energy from $\sim 26.5$ to $\sim 20.7 \mathrm{~nJ}$, when the HDF elongated from $\sim 1$ to $\sim 3 \mathrm{~m}$, owing to the increased absorption.

In theory, the signal induced saturation of the absorbing fiber should also influence the behavior of the setup. This can be found from our calculation in Fig. 2(c). In experiment, however, So far we did not observe the signal induced saturation of the absorbing fiber when the maximum pump power of $\sim 3.89 \mathrm{~W}$ is used. We attribute it to the large saturation threshold of our currently constructed NAbLM.
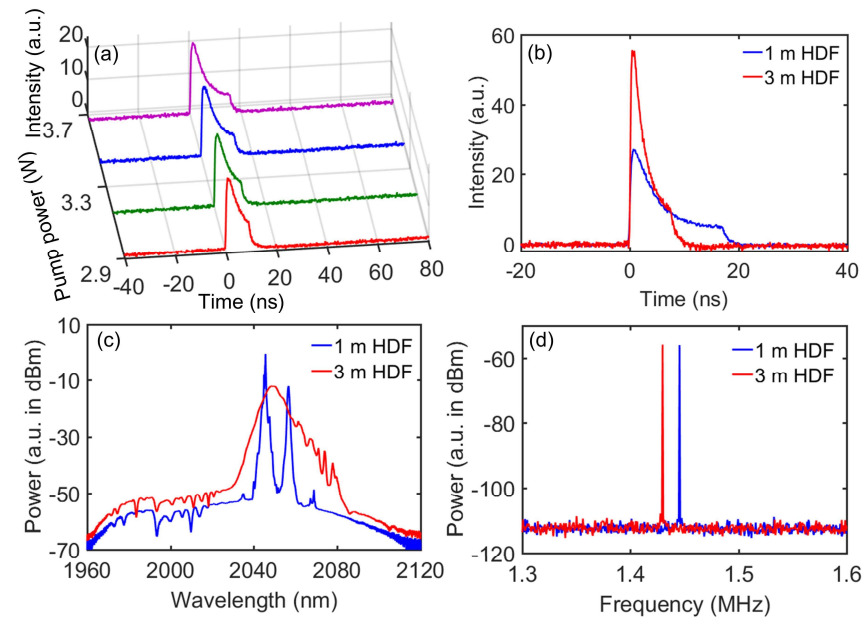

Fig. 3. (a) Evolution of single h-shaped pulse with the launched pump power when the HDF length was $\sim 1 \mathrm{~m}$; (b) single-pulse envelops and the corresponding (c) spectral profiles and (d) radio frequency traces with $\sim 1$ and $\sim 3 \mathrm{~m} \mathrm{HDF}$, respectively.

Figure 4 plots the average output power characteristics against the launched pump power at $\sim 1570.4 \mathrm{~nm}$, as well as the corresponding linear fits, with different lengths of unpumped HDF. As seen, all of them followed roughly linear evolutions. But the longer the incorporated unpumped HDF, the lower the emission power, i.e., the lower the slope efficiency. This results from that the reabsorption scales with the length of the unpumped HDF, which results in the net laser gain decreasing.

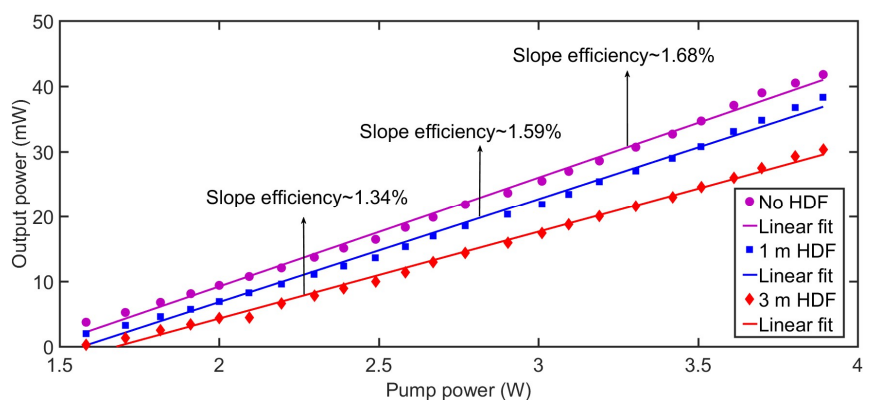

Fig. 4. Output average power versus $\sim 1570.4 \mathrm{~nm}$ pump power when the unpumped HDF lengths are $0, \sim 1$, and $\sim 3 \mathrm{~m}$, respectively.

We also attempted to check if the NAbLM-based HDF laser could produce other kinds of pulses. We removed all the normal-dispersion UHNA7 fiber and make the cavity all anomalous dispersion. Then, as shown in Fig. 5(a), the fibers used in the NAbLM included $\sim 5 \mathrm{~m}$ long high nonlinearity fiber (HNLF, NL1550-ZERO, Yangtze Optical Fibre and Cable Co., Ltd.), 1.6 $\mathrm{m} \mathrm{HDF}$, and the left SMF-28e fiber pieces. The total cavity length was reduced to $\sim 26.5 \mathrm{~m}$.

With such an all-anomalous NAbLM, the output characteristics from the HDF laser are depicted in Figs. 5 (b) trough 5(d). Figure 5(b) plots a typical spectrum peaking at $\sim 2058.22 \mathrm{~nm}$ with $3-\mathrm{dB}$ bandwidth of $\sim 15.53 \mathrm{~nm}$. We used the aforementioned photodetector and a $63 \mathrm{GHz}$ digital oscilloscope (DSO-X 96204Q, Keysight Technologies, Inc.) to check the pulse characteristics. Figure 5(c) plots a pulse envelop, showing no h-shaped characteristic. A captured pulse train is plotted in the inset. Figure 5(d) plots the RF spectrum around the fundamental pulse repetition rate of $\sim 7.765 \mathrm{MHz}$. It is a typical state of noise-like pulse (NLP) as Fig. 5(d) shows 
two featured broad subpeaks [14], [15]. This means that the NAbLM can still function well when the loop is not long and in a considerably different dispersion regime.
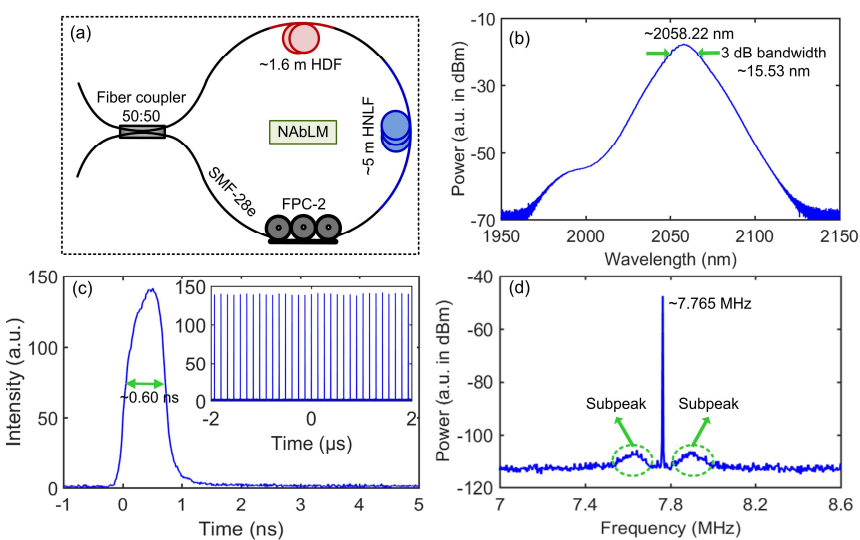

Fig. 5. (a) NAbLM with anomalous dispersion; (b) spectral profile; (c) pulse envelop (Inset: pulse train); (d) RF spectrum around the fundamental repetition rate.

To further check if other kind of pulses besides the NLP could be produced in the anomalous dispersion regime, we further made two different changes with the NAbLM. One was to further shorten it for weakening the effects related to cavity nonlinearity. The second was to lengthen it for greatly enhancing the overall cavity nonlinearity.

In experiment, however, we discovered that shortening the cavity was not a good option, which led to Q-switching rather than mode-locking. This results from the fact that, in the NAbLM, the used fiber absorber itself can also be seen as a type of SA, which has always been used for passive Q-switching [17-19]. Thus, to realize mode-locking we have to lengthen the cavity to eliminate the passive Q-switching [19]. As a consequence, if only for passive mode-locking purpose, the SA-incorporated NAbLM is appropriate for applications where the cavity roundtrip time is long enough. Certainly the exact length is determined by the used fiber absorber, as well as other parameters relating to the whole NAbLM. It can be expected that if the saturable fiber absorber is replaced with a non-saturable absorber, the NAbLM might be applicable in various circumstances. This is an interesting issue and deserved to be further explored.

As for the investigation here, then we took the second option. We lengthened the cavity by adding $\sim 100 \mathrm{~m} \mathrm{SMF-28e}$ fiber in the NAbLM as seen in Fig. 6(a). Through adjusting the FPCs, two notable, distinctly different pulse types could be achieved when the incident pump power was fixed. Comparatively, one exhibited high peak power and short duration, but the other exhibited low peak power and long duration. Figures 6(b) through (e) plot the emission characteristics with the same incident pump power of $\sim 3.89 \mathrm{~W}$.

Figure 6(b) shows the recorded pulse envelops. For the narrow pulse, its duration of $\sim 0.92 \mathrm{~ns}$ approaches the response time of the photodetector. For the wide pulse of duration of $\sim 5.88 \mathrm{~ns}$, it was further observed that the pulse duration was roughly proportional to the increase of pump power; meanwhile, the pulse peak widely spanned, as shown in the inset of Fig. 6(b). Thus, this pulse highly resembles the dissipative soliton resonance (DSR) pulse. We call it DSR-like pulse [5], considering that it was realized in the anomalous dispersion regime.

The RF characteristics further reveal that the narrow pulse should still be the NLP, since the subpeaks can still be noticed, as seen in Fig. 6(c). As for DSR-like pulse, however, as seen in Fig. 6(d) no subpeaks can be observed at the vicinity of the main RF peak, indicating that the DSR-like pulse should be noise-free.

Figure 6(e) plots their spectral profiles. Different to sharp peaks typically with net normal dispersion, here both pulses show wide and smooth spectral profiles. But the NLP has a much larger bandwidth $(\sim 30.39 \mathrm{~nm})$ than that of the DSR-like pulse $(\sim 12.60 \mathrm{~nm})$, probably due to the higher pulse peak power. The central wavelengths for the NLP and DSR-like pulse are $\sim 2036.92 \mathrm{~nm}$ and $\sim 2027.53 \mathrm{~nm}$, respectively. The DSR-like pulse also shows a higher slope efficiency than the NLP, as seen in Fig. 6(f).
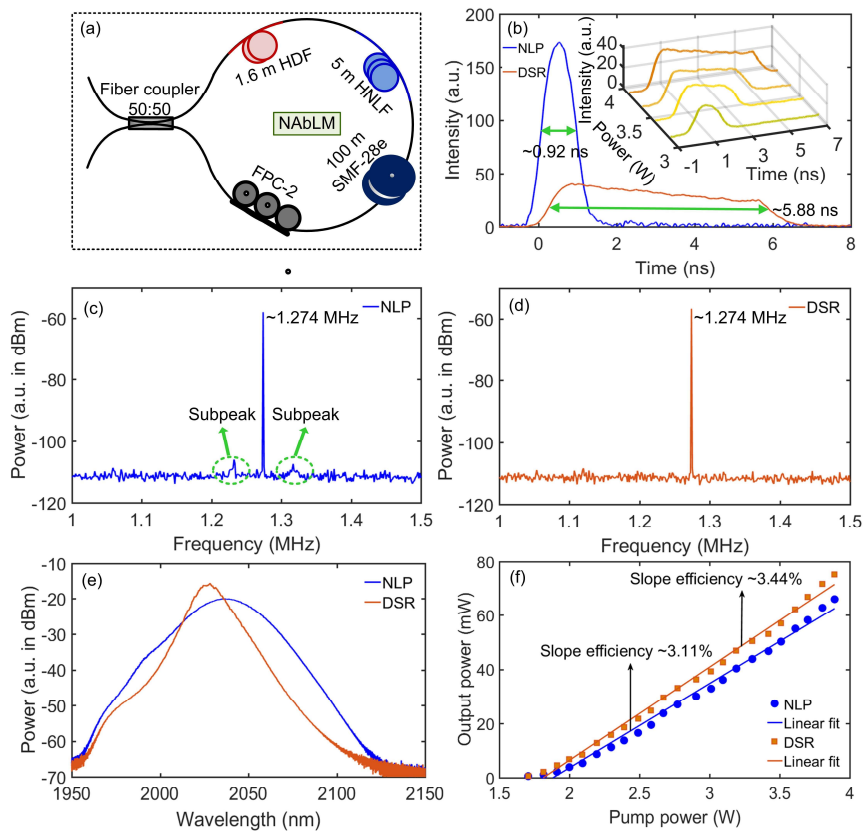

Fig. 6. (a) Lengthened NAbLM with anomalous dispersion; (b) pulse envelops, Inset: DSR-like pulse evolution with the incident pump power; (c) and (d) RF spectra; (e) optical spectra; (f) output power against the incident pump power.

The main mechanism for the pulse shape changes should be the PPC effect that directly relates to the intracavity nonlinearity. During the h-shaped pulse formation in Fig. 3, the incorporated long-piece of UHNA7 fiber resulted in considerable amount of accumulated nonlinearity. Thus, strong PPC effect was induced. In our case it led to the h-shaped pulse formation. Although this type of pulse intrinsically exhibits a tiny portion of unclamped leading edge, it is strongly shaped by the PPC effect across its most temporal span [4]. During the NLP generation in Fig. 5, however, the overall cavity nonlinearity was largely reduced due to the removal of UHNA7 fiber. As a consequence, no significant PPC effect took place, and only the NLP was observed. In Fig. 6, when we incorporated $\sim 100 \mathrm{~m} \mathrm{SMF-28e}$ fiber, the cavity nonlinearity was again greatly enhanced, leading to PPC effect under appropriate conditions. Further considering that it was in an anomalous dispersion regime, the DSR-like pulse was able to form, besides the NLP. 


\section{CONCLUSIONS}

In conclusion, we have constructed a type of HDF-based NAbLM. In theory, it shows enhancements in both extinction ratio and modulation depth, and can also achieve full switching between SA and RSA. It was then used in an HDF laser as an SA, enabling mode-locked operations in both net normal and all anomalous dispersion regimes. NAbLM can be used as an alternative in nonlinear optics, possessing some unique properties owing to the readily available equal-power interference.

\section{ACKNOWLEDGEMENT}

Mariusz Klimczak would like to thank the Fundacja na rzecz Nauki Polskiej (FNP) in scope of First TEAM/2016-1/1 (POIR.04.04.00-00-1D64/16); Lei Li and Luming Zhao would like to thank the Jiangsu Overseas Visiting Scholar Program for University Prominent Young \& Middle-aged Techers and Presidents and also the Priority Academic Program Development of Jiangsu Higher Education Institutions (PAPD).

\section{REFERENCES}

[1] M. G. Kane, I. Glesk, J. P. Sokoloff, and P. R. Prucnal, "Asymmetric optical loop mirror: analysis of an all-optical switch,” Appl. Opt., vol. 33, no. 29, pp. 6833-6842, 1994.

[2] N. J. Doran and D. Wood, "Nonlinear-optical loop mirror," Opt. Lett., vol 13, no. 1, pp., 56-58, 1988.

[3] M. E. Fermann, F. Haberl, M. Hofer, and H. Hochreiter, "Nonlinear amplifying loop mirror,” Opt. Lett., vol. 15, no. 13, pp. 752-754, 1990.

[4] J. Zhao, L. Li, L. Zhao, D. Tang, and D. Shen, "Cavity-

birefringence-dependent h-shaped pulse generation in a thulium-holmium doped fiber laser," Opt. Lett., vol. 43, no. 2, pp. 247-250, 2018.

[5] J. Zhao, L. Li, L. Zhao, D. Tang, and D. Shen, "Dissipative soliton resonances in a mode-locked holmium-doped fiber laser," IEEE Photon. Technol. Lett., vol. 30, no. 19, pp. 1699-1702, 2018.

[6] R. Liao, Y. Song, L. Chai, and M. Hu, "Pulse dynamics manipulation by the phase bias in a nonlinear fiber amplifying loop mirror," Opt. Express, vol. 27 , no. 10 , pp. 14705-14715, 2019

[7] G. K. Zhao, H. J. Chen, H. Z. Liu, W. C. Chen, A. P. Luo, X. B. Xing, H. Cui, Z. C. Luo, and W. C. Xu, "Coexistence of rectangular and Gaussian-shape noise-like pulses in a figure-eight fiber laser," Opt Express, vol. 26, no. 14, pp. 17804-17813, 2018.

[8] J. Zhao, J. Zhou, L.Li, L. Zhao, D. Tang, D. Shen, and L. Su, “Dissipative soliton resonance and its depression into burst-like emission in a holmium-doped fiber laser with large normal dispersion," Opt. Lett., vol. 44, no. 10 , pp. $2414-2417,2019$

[9] A. W. O'Neill and R. P. Webb, “All-optical loop mirror switch employing an asymmetric amplifier/attenuator combination," Electro. Lett., vol. 25, no. 24, pp. 2008-2009, 1990.

[10] J. Shi, Y. Li, S. Gao, Y. Pan, G. Wang, R. Ji, and W. Zhou, "All-polarization-maintaining figure-eight Er-fiber ultrafast laser with a bidirectional output coupler in the loss-imbalanced nonlinear optical loop mirror," Chin. Opt. Lett., vol. 16, no. 12, pp. 121404, 2018.

[11] M. D. Pelusi, Y. Matsui, and A. Suzuki, "Pedestal suppression from compressed femtosecond pulses using a nonlinear fiber loop mirror," IEEE J. Quantum Electron., vol. 35, no. 6, pp. 867-874, 1999.

[12] A.G. Striegler and B. Schmauss, "Extinction ratio improvement by an advanced NOLM setup,” IEEE Photon. Technol. Lett., vol. 18, no. 9, pp. 1058-1060, 2006

[13] G. P. Agrawal, Applications of Nonlinear Fiber Optics (2nd Edition), Academic Press, 2008.

[14] M. Michalska and J. Swiderski, "Noise-like pulse generation using polarization maintaining mode-locked thulium-doped fiber laser with nonlinear amplifying loop mirror,” IEEE Photon. J., vol. 11, no. 5, pp. $1504710,2019$.
[15] H. Xia, H. Li, G. Deng, J. Li, S. Zhang, and Y. Liu, "Compact noise-like pulse fiber laser and its application for supercontinuum generation in highly nonlinear fiber,” Appl. Opt., vol. 54, no. 32, pp. 9379-9384, 2015.

[16] J. Gene, S. K. Kim, and S. D. Lim, "Validity analysis of holmium-doped fiber as saturable absorber for passively Q-switched thulium-doped fiber laser,” J. Lightwave Technol., vol. 36, no. 11, pp. 2183-2187, 2018.

[17] L. A. M. Ya. E. Sadovnikova, V. A. Kamynin, A. S. Kurkov, O. I. Medvedkov, and A. V. Marakulin, "Q -switching of a thulium-doped fibre laser using a holmium-doped fibre saturable absorber," Quantum Electron., vol. 44, no. 1, pp. 4-6, 2014.

[18] M. Hu, B. He, H. Liu, Y. Yang, Y. Zheng, X. Chen, L. Zhang, and J. Zhou, "High-Peak Power, All-Fiber Passively Q-Switched Laser Using a Sm-Doped Fiber Saturable Absorber," J. Lightwave Technol., vol. 32, no. 14, pp. 2510-2515, 2014.

[19] A. E. Siegman, Lasers. Mill Valley, CA, USA: Univ. Sci., $1986 .$.

Junqing Zhao received the Doctor of Engineering degree in Optical Engineering from Shenzhen University, Shenzhen, China, in 2014, for research on pulsed fiber lasers. Since then, his research has covered device, system, and application aspects of fiber lasers and amplifiers, first with the Shenzhen Key Laboratory of Laser Engineering, Shenzhen University, China, and later with the Optoelectronics Research Centre (ORC), University of Southampton, U.K. $\mathrm{He}$ is currently with the Jiangsu Key Laboratory of Advanced Laser Materials and Devices, School of Physics and Electronic Engineering, Jiangsu Normal University, Xuzhou, China. He has published some 50 scientific articles. He is a Life Member of the Optical Society of America.

Jian Zhou is currently pursuing the Master's Degree at the Jiangsu Key Laboratory of Advanced Laser Materials and Devices, School of Physics and Electronic Engineering, Jiangsu Normal University. He is mainly studying on mode-locked fiber lasers, fiber amplifiers, and particular microfiber devices.

Yuyuan Jiang is currently pursuing the Master's Degree at the Jiangsu Key Laboratory of Advanced Laser Materials and Devices, School of Physics and Electronic Engineering, Jiangsu Normal University. She is mainly studying on fiber-based devices and systems.

Lei Li was born in 1983. He received his bachelor and master degree in optics from Shandong Normal University, Jinan, China. He received his Ph.D degree in optical engineering from Shandong University, Jinan, China, in 2013. His research filed focuses on the ultrafast fiber lasers, nonlinear optics and optoelectronics technology.

Deyuan Shen received the Ph.D. degree in physics from Shandong University, Jinan, China, in 1996. He subsequently joined the Institute for Laser Science, University of Electro-Communications, Tokyo, Japan, as a Postdoctoral Research Fellow, where he worked on laser-diode pumped high-power solid-state lasers. From 2000 to 2002, he was with the School of Electrical, and Electronic Engineering, Nanyang Technological University, Singapore, developing high-power visible and infrared solid-state lasers for industrial applications. He joined the Optoelectronics Research Centre (ORC), University of Southampton, Southampton, U.K., in 2002, and promoted to a Senior Research Fellow in 2005. His research activities at ORC included eye-safe high-power fiber lasers, fiber-bulk hybrid solid-state lasers, and novel fiber laser sources. His current research interests include power and brightness scaling of cladding pumped fiber lasers and amplifiers, nonlinear frequency conversion, and laser applications.

Andrey Komarov received the Bachelor Degree of Physics and Magister Degree of Physics from Novosibirsk State University (NSU), Russia, and the degree of Doctor of physical and mathematical sciences at Institute of Laser Physics, SB RAS), Russia. The main field of his scientific interests is nonlinear dynamics of ultrashort pulses in lasers and various nonlinear systems. A. Komarov has developed the theory of laser passive mode-locking which describes the quantization of radiation into identical dissipative solitons, multistability and multihysteresis phenomena, threshold self-start of passive mode-locking, and other effects. He is the specialist on numerical simulation of nonlinear optical systems. He is the author of 150 publications.

Lei Su received the B.Eng. and M.Eng. degrees from Xi' an Jiaotong University, Xi' an, China, and the Ph.D. degree from Nanyang Technological University, 
Singapore. He is a Lecturer in biomedical engineering with the School of Engineering and Materials Science, Queen Mary University of London, London, U.K.

Dingyuan Tang received the B.S. degree in physics from Wuhan University, in 1983, the M.S. degree in laser physics from the Shanghai Institute of Optics and Fine Mechanics, Chinese Academy of Science, in 1986, and the Ph.D. degree in physics from the Hannover University, Germany, in 1993. From 1993 to 1994, he worked as a scientific employee at the Physikalisch-Technische Budesanstalt, Braunschweig, Germany. From 1994 to 1997, he was a university Postdoctoral Research Fellow, and from 1997 to 1999, he was an Australian Research Council (ARC) Postdoctoral Research Fellow, both at the University of Queensland, Australia. From 1999 to 2000, he was a Research Fellow in the Optical Fiber Technology Center (OFTC), the University of Sydney, Australia. $\mathrm{He}$ is currently an Associate Professor in the School of Electrical and Electronic Engineering, Nanyang Technological University, Singapore, and a Special Professor at Jiangsu Normal University, China. Dr. Tang is a Member of Optical Society of America (OSA) and the Australian Optical Society (AOS).

Mariusz Klimczak obtained his M.Sc. and Ph.D. degrees at Warsaw University of Technology, Faculty of Electronics and Information Technology, in 2004 and 2010, respectively. His main research interests included spectroscopic characterization of dielectric optical materials activated with trivalent rare-earth ions, specifically in context of their short-wavelength emission properties under single-photon, as well as multi-photon or multi-ion excitation conditions. He joined Institute of High Pressure Physics of Polish Academy of Sciences in 2010, to investigate tunable semiconductor lasers and to conduct further research into laser diode-pumped devices. In 2013 he moved to the Institute of Electronic Materials Technology (ITME).

Luming Zhao received the B.Eng., and M.Eng. degrees from Tsinghua University, Beijing, China, and the Ph.D. degree from Nanyang Technological University, Singapore. He is currently a Full Professor with Jiangsu Normal University, Xuzhou, China. His research interests include ultrafast optics, fiber oscillators, fiber amplifiers, and soliton dynamics. 